\section{VIRTUAL REALITY THERAPY: AN EFFECTIVE TREATMENT FOR THE FEAR OF PUBLIC SPEAKING}

\author{
Max M. North, Sarah M. North, and Joseph R. Coble \\ Clark Atlanta University \\ Atlanta, Georgia, USA
}

Editor: Han Sieburg

\begin{abstract}
The major goal of this research was to investigate the efficacy of virtual reality therapy (VRT) in the treatment of the fear of public speaking. After an extensive two-stage screening process, sixteen subjects were selected from the pool. They were assigned to two treatment conditions: VRT $(\mathrm{N}=8)$ and comparison group $(\mathrm{N}=8)$. Fourteen subjects completed the study. The VRT group was exposed to the virtual reality public speaking scene while the comparison group was exposed to a trivial virtual reality scene and guided by the experimenters to manage their phobia either by using visualization techniques or selfexposure to the situation they feared. The VRT and comparison group sessions were conducted on an individual basis over a five week period. Two assessment measures were used in this study. The first measure used was the Attitude Towards Public Speaking (ATPS) Questionnaire. The second measure used was the eleven-point Subjective Units of Disturbance (SUD) scale. These measurements assessed the anxiety, avoidance, attitudes and disturbance associated with their fear of public speaking before and after treatments. In addition, objective measures such as heart rate was collected in each stage of the treatment.

Significant differences between the six subjects who completed the VRT sessions and comparison group were found on all measures. The VRT group showed significant improvement after five weeks of treatment. The comparison group did not show any meaningful changes. The authors concluded that VRT was successful in reducing the fear of the public speaking.
\end{abstract}

\section{Introduction}

The fear of speaking is often cited as the world's most common social phobia. This communication disorder is frequently identified among the top five most prevalent phobias. It does not appear to be limited by age, gender, economic or educational variables. Until this time, traditional treatment has included systematic desensitization, cognitive restructuring and skills building. Current advances made through the use of computer display technology and the work of researchers (authors) in Atlanta and Boston is responsible for the creation of virtual reality technology for use in treating the fear of public speaking and other psychological disorders. This research was conducted in the Virtual Reality Technology Laboratory at Clark Atlanta University (CAU) and with technical assistance of The Speech Improvement Company in Boston. To the best of our knowledge, this is the first known controlled study of the effectiveness of virtual reality therapy in the treatment of subjects who suffer from the fear of public speaking.

Research into this widespread phobia was conducted through the collaboration of CAU, the U.S. Army Research Laboratory and Boeing Computer Services, with special technical assistance from the Speech Improvement Company, Inc. With the assistance of the Speech Improvement Company, the research will have a positive impact on the clinical sessions of subjects suffering from the fear of public speaking. Additionally, the general population will benefit from the virtual reality research because the new technology provides greater access to a safe, confidential and economical approach to the treatment of psychological disorders.

The subjects' anxiety and avoidance behavior were interfering with their normal activities. They were unable to participate in the social gatherings, classes, or professional conferences. The symptoms experienced by the subjects during virtual realty therapy included an increase in heart rate, feeling a lump in the throat, dry mouth, sweaty palms, loss of balance, weakness in the knees, etc. These symptoms also appeared in the studies which dealt with the treatment for acrophobia (fear of heights), agoraphobia, and fear of flying.

Similar to our first known controlled studies of VRT, the study of the fear of public speaking indicated that VRT was very effective in reducing self-reported anxiety. The VRT treatment resulted in both a significant reduction in anxiety symptoms, as measured by SUD and ATPS, and the ability to face the phobic situations in the real world. At this time, several of the subjects can comfortably speak in front of a crowd with confidence.

The idea of using virtual reality technology to combat psychological disorders was first conceived in the HumanComputer Interaction Group at CAU in November 1992. Since then, the first known pilot studies in the use of virtual reality technologies in the treatment of agoraphobia have been conducted. This includes an investigation of the use of virtual reality for specific phobias: fear of flying [North \& North 1994], fear of heights [Williford, Hodges, North \& North, 1993; Rothbaum, Hodges, Opdyke, Kooper, Williford \& North, 1995; North, North \& Coble 1995b] and fear of being in certain situations such as a dark barn, an enclosed bridge over a river, and the presence of an animal, such as a black cat in a dark room [North, North \& Coble 1995a; 1996a; 1996b]. Other researchers have realized the potential of virtual reality in the treatment of psychological disorders. These contemporary research activities have established a new paradigm that is attracting serious scientists from the computer science, psychology, and medical fields. The present study sought to extend the previous work to include a larger number of subjects and a wider range of psychological disorders. 


\section{Method}

\subsection{SUBJECTS}

Subjects for the research were recruited from introductory psychology classes at Clark Atlanta University. A group of thirty-five undergraduates underwent and extensive two-stage screening process to ensure that they were suffering from the fear of public speaking and had no other serious physical or psychological conditions. The participation of all subjects was strictly voluntary.

The first stage of the screening process consisted of a set of questionnaires administered in the psychology classes. The questionnaires contained items that screened students for the fear of public speaking consistent with the diagnosis of a specific phobia [American Psychiatric Association, 1994]. The questionnaires excluded subjects with panic conditions and other specific disorders, including substance disorders, major medical illness including thyroid disease, and the use of medication with significant psychotropic or physiologic effects. Additional screening criteria included in the questionnaires was symptom duration for at least one year and strong motivation for overcoming a phobia.

The second phase of the screening process involved a more stringent diagnostic procedure. It included a clinical interview and completion of the modified Attitude Towards Public Speaking Questionnaire [Abelson and Curtis, 1989]. Subjects having symptoms limited to the fear of public speaking, a social phobia and consistent with the diagnosis of a specific phobia were included in the study. During this phase, subjects also completed a questionnaire regarding various demographic variables, which included sex, geographical region, rural vs. urban, socioeconomic status, experience with computers, computer games and/or virtual reality.

The remaining subjects were assigned to two treatment conditions: virtual reality therapy (VRT) $(\mathrm{N}=8)$, and a comparison group $(\mathrm{N}=8)$. The two groups were as closely matched as possible with respect to demographic and severity of symptoms. In order to minimize the confounding variable of treatment expectancy, informed consent for treatment statements were obtained after the group assignment. After the first set of assigned measures, the comparison group subjects were informed that they would undergo repeated testing at the end of the five weeks. They were exposed to the trivial virtual reality scenes and advised by the experimenters to manage their fear and expose themselves to the situations they were avoiding. This approach was used to offset the placebo effects. The comparison group was not treated with a systematic treatment program. All subjects in the two groups were asked not to communicate with other subjects and not to self-treat with any relaxation after exposure to the phobic situation. All subjects were asked to keep a diary of any new medication regimens, including over-the-counter medications, caffeine use, significant stresses, and new illness and/or treatment.

\subsection{ASSESSMENT MEASURES}

The screening questionnaires used in this study assessed several inclusion and exclusion criteria. DSM-IV criteria for specific phobia (i.e., fear of public speaking, avoidance of feared situations, belief that the fear is excessive, interference from fear), desire for treatment, desire to participate in a treatment study, presence of panic attacks, history of panic attacks, and presence of claustrophobia were assessed by this instrument.

This Attitude Towards Public Speaking Questionnaire (ATPS) contains six items that assess attitudes toward the fear of public speaking. It is adapted from Abelson and Curtis [1989] with modifications that relate to the fear of public speaking. The following dimensions, rated on a 0-10 semantic differential scale: good-bad, awful-nice, pleasantunpleasant, safe-dangerous, threatening-nonthreatening, and harmful-harmless are included.

The Fear Questionnaire was constructed for use in this study. The Marks and Mathew' [1979] Fear Questionnaire which assesses the degree of distress experienced from the fear of public speaking was also used. The four situations that subjects were exposed to were speaking in an auditorium without any audience; speaking in an auditorium with audience; speaking to an audience who talked to each other and did not pay attention to the speaker; or speaking to an audience who laughed at them. Each of these categories was varied by the size of the audience, ranging from 0 to 100. The situations were rank ordered and rated based on the level of discomfort produced. This determined the most appropriate use of the VRT.

Reactions according to a modified version of the Subjective Units of Disturbance (SUDS) scale were collected every few minutes during exposure on a 0 (no discomfort) to 10 (panic-level anxiety) scale [Wolpe, 1969]. This is a very quick and simple measure of anxiety. The method has been used widely and been shown to correlate well with objective physiological measures of anxiety [Thyer et al, 1984].

The instruments used in the pilot studies were of a selfreporting nature. The validity of the data depended on the attitude, honesty, and accuracy of the participants' responses. Current literature regarding virtual reality research recommends the use of physiological data (e.g., heart rate, blood pressure, galvanic skin response, EEG/EMG, etc.) to measure the sense of presence, perception of physically being in a virtual world. For more information on sense of presence in virtual reality, readers may refer to the references listed in this article. For instance, Barfield addresses this issue and writes that techniques which measure sense of presence typically and primarily involve subjective assessment, which is most useful for initial exploration and hypothesis generation [Barfield and Weghorst, 1993]. Barfield's hypothesis is that as sense of presence increases within a virtual environment, the participant should experience physiological changes that can be measured and analyzed to determine the optimal sense of 
presence with a minimal visual presentation of virtual reality scenes and objects. The authors planned to use physiometric indicators of sense of presence which are readily quantifiable. The physiological instruments are normally used to measure various changes in subjects' bodily functions. The changes in bodily functions might possibly be indicators of physiological arousal purportedly linked to virtual reality sense of presence and performance. For the purpose of monitoring the subjects, researchers collected only the heart rate of the subjects in this current experiment.

\subsection{APPARATUS}

Hardware for this study consisted of a Pentium-based ${ }^{\mathrm{TM}}$ computer (100 MHZ), Head-Mounted Display and HeadTracker (Virtual $\mathrm{I} / \mathrm{O}^{\mathrm{TM}}$ ) so that the use could interact with objects in the virtual world. Modeling was done by using VREAM $^{\mathrm{TM}}$ Virtual Reality Development Software Package and Libraries (VRCreator ${ }^{\mathrm{TM}}$ ) to create virtual reality scenes from the models which represented a customized hierarchy of fear producing situations.

\subsection{VIRTUAL REALITY SCENE}

Modeling was done by the VREAM ${ }^{\mathrm{TM}}$ Company under the direction of the researchers. A model of auditorium located in the CAU Research Science Building was created. The virtual auditorium is 48 feet wide, 100 feet long and 55 feet high. The seating area has three sections of chairs and can accommodate over 100 people. (See Figure 1 on the CDROM.) The software was designed to allow the experimenter to bring the audience into the auditorium one by one and then five at a time until the auditorium was filled. Several audio clips were programmed to respond to the speaker or create a variety of situations for them to experience, such as laughing, making comments, encouraging the speaker to speak clearer or louder, ignoring the speaker and holding conversations with others, and clapping hands at the end or during the session. Specialized features created for the facility included a virtual wooden podium with a speaker's stand. An amplifier with direct connection to the virtual reality software and hardware were used in the therapy sessions. This enabled the subjects to hear the echo of their voices. Simulations of the real echo in the auditorium were created by a headphone attached to the head-mounted display. The treatment schedule consisted of five weekly sessions. The sessions lasted 10 to 20 minutes.

\subsection{TREATMENT PROCEDURE}

The treatment schedule consisted of five weekly sessions for the VRT and comparison groups. The first two sessions lasted 10 to 15 minutes and each of the three subsequent sessions lasted 15 to 20 minutes. VRT experiments were conducted in the Virtual Reality
Technology Laboratory on the CAU campus. Comparison group experiments were partially conducted in the CAU Psychology Department and Virtual Reality Technology Laboratory. The comparison subjects reported for the selfrated treatment expectancy and the repeated assessment in the Psychology Department.

In the first treatment session, the VRT subjects were asked to rank order a list of public speaking situations that induce fear, based on the degree of anxiety they arouse. These hierarchies were used later for the VRT [Pendleton and Higgins, 1983]. During the VRT subjects' and comparison subjects' first session, they were individually familiarized with the virtual reality equipment and given several virtual reality demonstrations.

For the VRT subjects' subsequent sessions, individually therapy was conducted in a standard format. The computer program designed for VRT generated a standard hierarchy of fearful public speaking situations to be presented to the VRT group. Assessment measures were administered under blind conditions and in a standard order.

To assess the subjects, each of the two subjects groups were administered the ATPS and the modified SUD as pretreatment, and post-treatment.

\subsection{SUBJECT RISK}

The subject risk in this project was minimal. While there are some potential risks associated with virtual environment technology, as pointed out by Stanny [1995], definite steps were taken in this project to minimize these risks. According to Stanny, subjects at risk for psychological harm are primarily those who suffer from panic attacks, those with serious medical problems such as heart disease or epilepsy, and those who are or have recently been taking drugs with major physiological or psychological effects. As is previously stated, questions regarding these situations were asked as a part of the screening process, and persons with these characteristics were excluded from serving as subjects in this project. The project researchers are also aware that some people experience symptoms ranging from headache to epileptic seizure when exposed to visual stimuli, which flicker at $8-12 \mathrm{~Hz}$. This project did not use any frame update rates in this range. Furthermore, subjects were closely observed by experimenters at all times. If there had been any evidence of significant physical or psychological distress, the subject and the experimenter had the ability to quickly terminate the virtual environment session. Given these safeguards, and the fact that no subjects have experienced harm in the previous virtual environment studies at CAU, the researchers feel confident in saying that patient risk in this study was minimal and acceptable.

\subsection{CONSENT}

Written informed consent was obtained (1) prior to initial behavioral testing and (2) prior to beginning of treatment after the experimental procedures had been fully 
explained. Signed consent forms for all subjects were kept on secured file.

\section{Results}

Means and standard deviations of ATPS and SUD Pretreatment and Post-treatment scores of VRT and comparison group subjects were calculated. The results and analyses of the assessment of before (base line) and after treatment are presented in the table of Figure 2. The strong correspondence between scores on the two instruments provides a good validity check, since the tests measures subjective discomfort on a 11-point scale, with the SUD being simply a rating of general discomfort and the ATPS measures discomfort on six emotional dimensions.

Comparisons of relevant sets of group means were performed using t-tests. No significant differences were seen between the pre-test scores of the two groups on their instrument, which indicated that the two groups were well matched as to initial severity of discomfort. There were also no significant difference between the pre-test and posttest scores of the comparison group on either test. This demonstrated that the severity of symptoms did not spontaneously change over the course of the experiment. Significant differences were found between the pre-test and post-test scores of the VRT group on both instruments, and between the post-test scores of the two groups on both tests. These means and t-test results indicate that the post-test scores of the VRT group were significantly lower than the pre-test scores and the post-test scores of the comparison group. This implies a reduction in the fear of public speaking symptoms as a result of the VRT treatment.

An examination of the correlation and the corresponding probabilities indicates that there was a significant relationship at the .05 level between the heart rate recorded and measure of subject unit of disturbance (SUD) scale. A Pearson Product-Moment Correlation coefficient $(r$ ) was used to measure the strength of the relationship $(\mathrm{r}=0.414, \mathrm{p}=0.05)$.

Although somewhat limited, the present results are definitely important. They attest to the sense of presence experienced by subjects in the virtual environment. The degree of anxiety and habituation observed would not have occurred if the subjects had not been immersed in the virtual environment. The high SUD scores obtained during early training sessions indicate that the anxiety levels of subjects were raised through exposure to the virtual environments. The steady reduction in SUD scores across the training sessions indicate habituation (reduction in anxiety responses) as a result of the VRT treatment. With further research, the researchers believe that VRT may prove to be a cost-and- time-effective alternative to the treatment of phobic

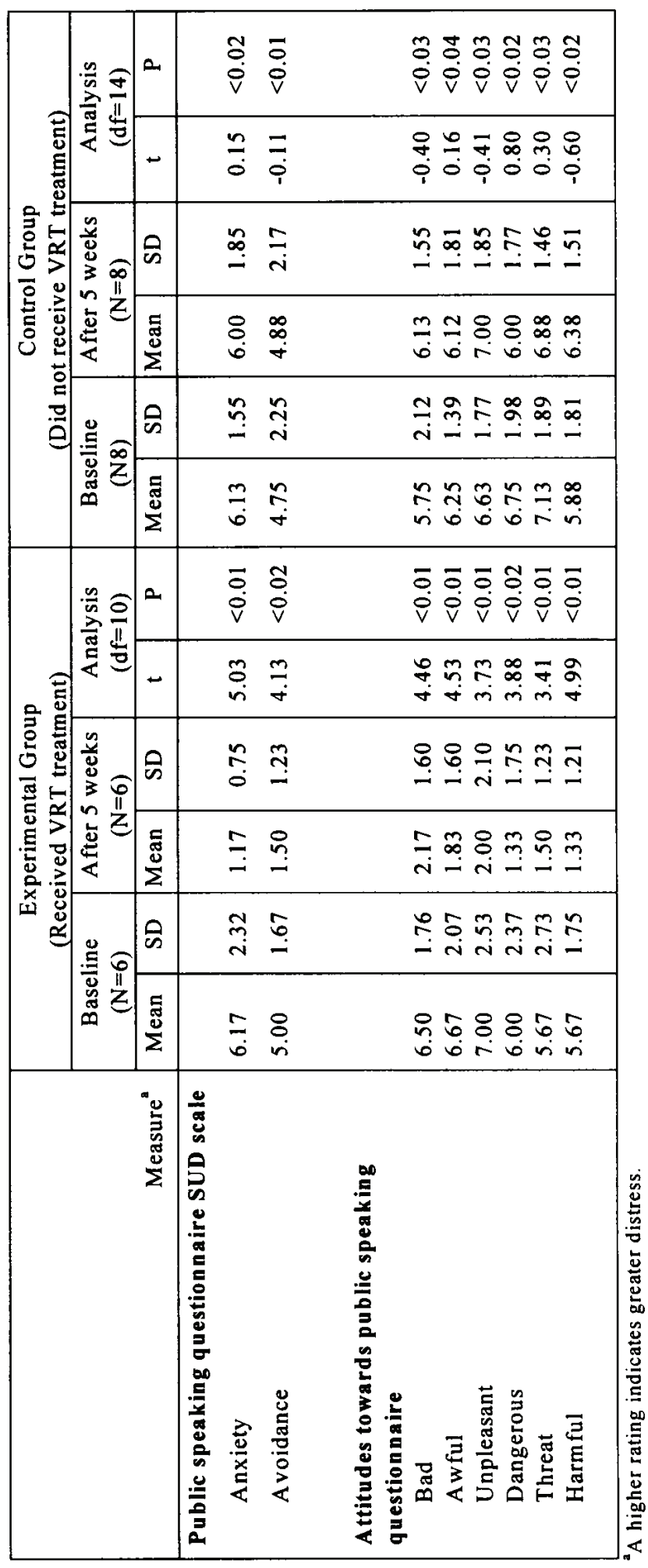

disorders. 


\section{Conclusion}

The symptoms experienced by the subjects during virtual reality therapy mirror those, which most speakers experience during a speech to a town meeting or presentation at a business conference. They include an increase in hear rate, lump in the throat, dry mouth, sweaty palms, loss of balance, weakness in the knees, etc.

It is apparent that the increase of anxiety at the beginning of each level and session and steady reduction after the subject spent some time in the virtual scene, attest to sense of virtual presence. This pattern also appeared in research studies with other phobic situations and subjects. The subject definitely became immersed in the virtual scene to the extend that they had to grasp a hand rail in the laboratory to avoid falling and to maintain their balance while they were in the virtual environment. [North, North and Coble, 1995a; 1995b; 1996].

It is also not unusual for longer exposure to virtual reality scenes to cause simulator sickness. This is caused by discrepancies between visual and kinetic perceptions; it is similar to motion sickness [Pausch et al., 1992]. Extended exposure to virtual reality may also cause physical and psychological injury. With these circumstances in mind, therapy sessions were limited to 10-20 minutes. This schedule was adopted to avoid any physical and emotional discomfort the subject might incur.

This study of the fear of public speaking indicates that VRT is very effective in reducing self-reported anxiety. The virtual reality therapy treatment results in both a significant reduction of anxiety symptoms (SUD and ATPS measurements) and the ability to face the phobic situations in the real world. After the treatment, subjects report that they have the ability to speak comfortably in front of a crowd with greater confidence.

This research project has the potential of significantly increasing the range of the psychological disorders that can be treated with VRT and, in turn, enhancing the significant of the findings.

\section{Discussion}

Although the results of the above-mentioned research are very impressive, additional research in needed to conduct a more thorough investigation of this approach with other psychological disorders (e.g., PTSD, ADD, etc.). There is also a need to conduct extensive investigation of virtual reality in the treatment of psychological disorders by using methods that allow both objective and subjective measurements of anxiety to insure the validity of this research. Furthermore, if the funding are sufficient and remain constant, the researchers hope to investigate the influence of subject variables (demographic and personality characteristics) on the effectiveness of VRT. They also hope to include an imaginal systematic desensitization (conventional therapy) group in addition to the comparison and control groups used in current or previous studies.
One possible criticism of the results of VRT studies at CAU and elsewhere is that the measures of anxiety involved have been primarily, and often exclusively, subjective. There is evidence to suggest that subjective measures of anxiety (more specifically, ratings on the Subjective Units of Disturbance scale) have a high correlation with objective measures of anxiety [Thyer et al, 1984]. This evidence was specifically evaluated in this study, where simultaneous subjective and objective measures of anxiety were obtained.

The current plan is to expand the research to a wider population and to extend the test site at the Virtual Reality Technology Laboratory in Atlanta to the Speech Improvement Company offices in Boston. This will allow the first known application of VRT technology to be held in a non-laboratory controlled setting with measurement in the real world.

\section{Acknowledgments}

This research project was sponsored by a grant from Boeing Computer Services (Virtual Systems Department), partially supported by U.S. Army Center of Excellence in Information Science under contract number DAAL03-92-60377 and The Speech Improvement Company. The views contained in this document are those of the authors and should not be interpreted as representing the official policies of the U.S. Government, either expressed or implied.

\section{REFERENCES}

Abelson, J. L. and Curtis, G. C. (1989). Cardiac and neuroendocrine responses to exposure therapy in height phobias: Desynchrony within the physiological response system. Behavior Research and Therapy, 27, pp. 561-565.

American Psychiatric Association: Diagnostic and Statistical Manual of Mental Disorders, 4th Edition. Washington, D.C., American Psychiatric Association (1994).

Barfield, W., and Weghorst, S. (1994). The sense of presence within virtual environments: A conceptual framework. In Human-Computer Interaction: Software and Hardware Interfaces. G. Salvendy and M. Smith (Eds). Elsevier Publisher, pp. 699-704.

Marks, I. M. And Mathews, A. M. (1979). Brief standard self-rating for phobic patients. Behavior Research and Therapy, 17, pp. 263-267.

North, M. M. and North, S. M. (1994). Virtual environment and psychological disorders. Electronic Journal of Virtual Culture, 2(4), pp. 37-42.

North, M. M., North, S. M., and Coble, J. R. (1995a). Effectiveness of virtual environment desensitization in the treatment of agoraphobia. International Journal of Virtual Reality, 1(2), pp. 25-34.

North, M. M., North, S. M. and Coble, J. R. (1995b). A virtual reality application in the treatment of psychological disorders. Journal of Medicine and Virtual Reality, 1(2), pp. 28-32. 
North, M. M., North, S. M. and Coble, J. R. (1996a). Effectiveness of virtual reality environment desensitization in the treatment of psychological disorders. PRESENCE: Teleoperators and Virtual Environments, 5(3), pp. 345-352.

North, M. M., North, S. M. and Coble, J. R. (1996b). Virtual Reality Therapy: An innovative paradigm. IPI Press: Colorado Springs.

Rothbaum, B. O., Hodges, L. F., Kooper, R., Opdyke, D., Williford, J. S., and North, M. M. (1995). Effectiveness of virtual reality graded exposure in the treatment of acrophobia. American Journal of Psychiatry, 152(4), pp. 626-628.

Thyer, B. A., Papsdorf, J. D., Davis, R., and Vallecorsa, S. (1984). Autonomic correlates of the subjective anxiety scale. Journal of Behavior Therapy and Experimental Psychiatry, 15, pp. 13-17.

Williford, J. S., Hodges, L. F., North, M. M. \& North, S. M. (1993). Relative effectiveness of virtual environment desensitization and imaginal desensitization in the treatment of acrophobia. Proceedings of Graphics Interface '93 Conference, p. 162.

Wolpe, J. (1969). The Practice of Behavior Therapy. Pergamon Press: New York.

\section{BIOGRAPHIES}

Max M. North, Ph.D. is a tenured Associate Professor in the Computer Information Science Department and Director of the Human-Computer Interaction Group \& Virtual Reality Technology Laboratory at Clark Atlanta University. He is the author of many articles in the field of computing and psychology and the recipient of an award from Sigma Xi, the Scientific Research Society. Dr. North's field of research is human-computer interaction. Specifically, his work focuses on studying "sense of presence" in virtual environments and designing virtual environments to study human-spatial perception and performance behavior in virtual environments. Dr. North's major contribution to the scientific community is his discovery and continuous strong research activities in the innovative area of virtual reality therapy which has received international attention and coverage in the scientific community and the popular media, including the New York Times, U.S. News and World Report, PBS, NBC, ABC.

\section{Contact Information:}

Max M. North, Ph.D.

Virtual Reality Technology Laboratory

Clark Atlanta University

Atlanta, GA 30314

Phone: 1-404-880-6942

FAX: 1-404-880-6963

Email: max@acm.org

URL: http://www.a2zsol.com/members/virtualreality
Sarah M. North, Ed.D. is a tenured Assistant Professor in the Computer and Information Science Department at Clark Atlanta University. She is Co-Director of the Human-Computer Interaction Group and Virtual Reality Technology Laboratory. She is the author of several articles in the field of computing and psychology. Professor North's field of research is cognitive science and humancomputer interaction. Essentially her work emphasizes the effectiveness of virtual reality in the motivational processes of learners. Professor North's current projects include 1) the study of the effectiveness of virtual environments for improving and maintaining the intrinsic motivation of learners, and 2) the development of better information visualization tools to support the characteristics of the human cognitive process. Her research activities have been reported in the public news media, including Atlanta Computer Currents and U.S. News and World Reports.

\section{Contact Information:}

Virtual Reality Technology Laboratory

Clark Atlanta University

Atlanta, GA 30314

Phone: 1-404-880-6957

Email: Sarah@acm.org

Joseph R. Coble, Ph.D. is a tenured Professor in the psychology department at Clark Atlanta University. He has been one of the most active members of the HumanComputer Interaction and Virtual Reality Technology Laboratory research team, publishing several papers in the field of psychology and computing. Dr. Coble's research activities in collaboration with Dr. North and Prof. North have been reported by several media. Dr. Coble received his training as a research psychologist from the psychobiology program at Florida State University with a masters in human vision and a doctorate in behavioral genetics.

Dr. Coble is basically a self-taught computer expert. His computer work has been primarily in the area of computer assisted instruction. $\mathrm{He}$ has been actively involved with the Clark Atlanta University School of Education in the development of several minority science improvement program grants which help to teach computer assisted instruction techniques to minority college faculty.

\section{Contact Information:}

Prof. Joseph R. Coble

Psychology Department

Clark Atlanta University

Atlanta, GA 30314

Phone: 1-404-880-8259

Email: jrcoble@prodogy.net 